\title{
Associação entre qualidade do sono e doenças cardiometabólicas de pacientes da Atenção Primária à Saúde
}

\author{
Association between sleep quality and cardiometabolic diseases of primary care \\ system patients
}

\section{AUTORES \\ Suelen Jane Ricardo ${ }^{1}$ (D) \\ Monique Yndawe Castanho de Araujo ${ }^{1}$ \\ Alessandra Madia Mantovani ${ }^{2}$ (D) \\ Lionai Lima dos Santos ${ }^{1}$ (D) \\ Bruna Camilo Turi ${ }^{2}$ (D) \\ Dayane Cristina Queiroz ${ }^{1}$ (D) \\ Jamile Sanches Codogno ${ }^{1,2}$ (iD \\ 1 Universidade Estadual Paulista, Departamento de Fisioterapia, Presidente Prudente, São Paulo, Brasil. 2 Universidade Estadual Paulista, Departamento de Ciências da Motricidade, Rio Claro, São Paulo, Brasil.}

\section{CONTATO}

Suelen Jane Ricardo

suel_jane@botmail.com

Rua Roberto Simonsen, 305, Centro Educacional, Presidente Prudente, São Paulo, Brasil. CEP: 19060-900.

DOI

$10.12820 /$ rbafs. $24 \mathrm{e} 0087$

\begin{abstract}
RESUMO
A privação do sono está relacionada ao desenvolvimento de doenças cardiometabólicas. O objetivo deste estudo foi analisar a associação entre qualidade do sono e ocorrência de doenças cardiometabólicas em adultos atendidos na Atenção Primária à Saúde. Estudo longitudinal composto por 169 adultos (68,6\% do sexo feminino), média de idade 61,80 (desvio padrão $=8,80$ ), acompanhados por 30 meses. A presença das doenças hipertensão arterial, dislipidemia, diabetes mellitus e ocorrência de infarto e a qualidade do sono foram mensuradas por meio de questionários. Os participantes foram divididos em grupos de acordo com a qualidade do sono (adequada/inadequada). Para verificar a diferença de idade, classe econômica, atividade física habitual e circunferência de cintura, entre os grupos a e b, utilizou-se o teste $t$ de Student para amostras independentes. Para comparar a proporção das doenças (hipertensão arterial, infarto, diabetes e dislipidemias) entre os grupos, utilizou-se o teste Qui-quadrado com correção de Yates. A regressão logística binária foi utilizada para testar a magnitude de tais associações, ajustadas por sexo, idade, atividade física, obesidade abdominal, etilismo e tabagismo. As análises estatísticas foram realizadas no software BioEstat (versão 5.0) e o nível de significância adotado foi de $\mathrm{p}<0,05$. Verificou-se que 83,4\% dos adultos reportaram ao menos uma alteração na qualidade do sono e os que apresentaram inadequada qualidade do sono tiveram 3,4 vezes mais chances de reportar diabetes $(\mathrm{OR}=3,47$; IC95\%: 1,06-11,30), comparados aos com o sono adequado. Inadequada qualidade do sono parece contribuir para o aumento da ocorrência de diabetes mellitus, independente do tempo de prática de atividade física e a obesidade abdominal.
\end{abstract}

Palavras-chave: Sono; Doenças cardiovasculares; Doenças metabólicas; Atividade motora.

\section{ABSTRACT}

Sleep deprivation is related to the development of cardiometabolic diseases. The aim of this study was to analyze the association between sleep quality and occurrence of cardiometabolic diseases in adults attending Primary Health Care. Longitudinal study of 169 adults (68.6\% female), mean age 61.80 (deviation= 8.80), followed for 30 months. The presence of the diseases hypertension, dyslipidemia, diabetes mellitus and occurrence of infarction and the quality of sleep were measured using questionnaires. Participants were divided into groups according to sleep quality (adequate / inadequate). To verify the difference in age, economic class, habitual physical activity and waist circumference, between groups a and b, the Student's t test for independent samples was used. To compare the proportion of diseases (hypertension, heart attack, diabetes, and dyslipidemia) between the groups, the chi-square test with Yates correction was used. Binary logistic regression was used to test the magnitude of such associations, adjusted for gender, age, physical activity, abdominal obesity, alcoholism and smoking. Statistical analyzes were performed using BioEstat software (version 5.0) and the significance level adopted was $p<0.05 .83 .4 \%$ of adults reported at least one change in sleep quality and those with inadequate sleep quality were 3.4 times more likely to report diabetes $(O R=3.47$; $95 \%$ CI: 1.06 -11.30) compared to those with adequate sleep. Inadequate sleep quality seems to contribute to the increased occurrence of diabetes mellitus, regardless of the length of physical activity and abdominal obesity.

Keywords: Sleep; Cardiovascular diseases; Metabolic diseases; Motor activity.

\section{Introdução}

O sono é responsável por reestabelecer funções do corpo e melhorar a saúde ${ }^{1,2}$. Por outro lado, a privação do mesmo impacta negativamente na saúde cognitiva e física, com prejuízos na memória, atenção e habilidades motoras de modo a contribuir para má qualidade de vida $^{1}$. Na Holanda, 43,2\% dos adultos relataram sono insuficiente, e $32,1 \%$ possuíam algum distúrbio relacionado à inadequada qualidade do sono (IQS) ${ }^{3}$. A inadequada qualidade no sono na Austrália é algo comum, 
afetando cerca de $45,0 \%$ dos adultos ${ }^{4}$. Estudo nacional identificou que $76 \%$ dos adultos no Brasil apresentou pelo menos um problema relacionado ao sono ${ }^{5}$.

Tem sido relatado na literatura que as pessoas que não dormem a quantidade de sono recomendada estão mais expostas ao risco de doenças cardiometabólicas ${ }^{6,7}$. A presença de IQS está associada a hospitalizações e maior procura por atendimentos médicos ${ }^{8}$. Doenças relacionadas à IQS têm impactos negativos nos custos com serviços de saúde $e^{8,9,10}$. Por exemplo, na Polônia, os custos totais com diabetes mellitus em 2009 foram de 654 milhões de euros ${ }^{9}$. No Brasil, as internações de adultos relacionadas à diabetes no ano de 2014 representaram custos de aproximadamente 1093,75 milhões de reais ${ }^{10}$.

Estudos que analisam a relação entre qualidade do sono (QS) e diversas doenças existem em âmbito mundial $23,6,7,9$. Entretanto, no Brasil a relação entre qualidade do sono adequada ou inadequada com doenças cardiometabólicas e os custos relacionados a tais doenças ${ }^{13,14}$ foi pouco estudada. Ademais, não se deve desconsiderar o fato que tanto a piora na $\mathrm{QS}$ como o desenvolvimento de doenças cardiometabólicas são desfechos fortemente afetados por variáveis como, por exemplo, inatividade física e obesidade ${ }^{13}$. Destaca-se a importância da prática de atividade física como instrumento de prevenção de doenças relacionadas a IQS, pois observou-se que IQS combinada com baixa prática de atividade física está associada a maiores chances de resistência a insulina em comparação com pessoas com adequada qualidade do sono (AQS) e que praticam atividade física ${ }^{15}$.

No contexto apresentado o estudo teve como objetivo verificar se há associação entre QS e ocorrência de doenças cardiometabólicas entre adultos atendidos na atenção primária.

\section{Métodos}

Trata-se de um estudo transversal que avaliou usuários do Sistema Único de Saúde (SUS) da cidade de Presidente Prudente, interior do Estado de São Paulo (população estimada em 225.271 habitantes e Índice de Desenvolvimento Humano - IDH de 0,806$)^{16}$. Este estudo foi desenvolvido com pacientes cadastrados nas Unidades Básicas de Saúde (UBS) do município. Os protocolos da investigação foram aprovados pelo Comitê de Ética em Pesquisa da Universidade Estadual Paulista Campus de Presidente Prudente (Processo no 241.291-2013).

O cálculo amostral levou em consideração que $60 \%$ da população brasileira é atendida exclusivamente pelo sistema público de saúde ${ }^{17}$, um erro amostral de
5 pontos percentuais, intervalo de confiança de $95 \%$ (IC95\%), acréscimo de 40\% para compensar perdas e recusas. Com base nesses parâmetros, estimou-se amostra de 518 adultos.

Inicialmente os avaliadores ficaram na UBS em um período de quatro semanas convidando os pacientes para participarem do estudo, mediante ao interesse, responderam os questionários e fizeram a avaliação os pacientes que se enquadraram nos seguintes critérios de inclusão: a) Ter ao menos uma consulta na UBS nos seis meses anteriores a data da primeira coleta (março de 2014), como forma de verificar se o usuário estava ativo na UBS; b) idade igual ou superior a 50 anos e; c) ter assinado o Termo de Consentimento Livre e Esclarecido. Na presente pesquisa foram incluídos apenas os pacientes que participaram das cinco avaliações $(\mathrm{n}=$ 169). Os critérios de exclusão foram: não ter participado em pelo menos uma das avaliações do estudo.

A coleta de dados foi realizada nos anos de 2014 a 2016, no período de setembro a março, de cada ano, por avaliadores treinados e que participavam do Grupo de Estudo em Saúde, Atividade Física e Economia (GESAFE). Em cada coleta foram verificadas informações referentes aos seis meses anteriores, totalizando 30 meses de acompanhamento dos participantes.

Para identificar a presença de doenças crônicas foi utilizado o autorrelato de morbidades, assim como realizado anteriormente em mulheres brasileiras de 21 a $81 \operatorname{anos}^{18}$. Neste instrumento são coletadas informações sobre a presença de doenças (hipertensão arterial, diabetes mellitus, dislipidemia e ocorrência de infarto), tempo de diagnóstico e uso de medicamentos. Para fins de análise, foi adotado o número de vezes em que o participante reportou a presença de tais doenças ao longo dos anos de seguimento do estudo (oscilando de 0 [não reportou] a 5 [desde as medidas de linha de base]). Para fins de análise, essa variável foi recategorizada em: ausência da doença (escore zero) e presença da doença (escore variando de 1 a 5).

A medida da QS foi feita por meio do Mini-sleep Questionnaire, instrumento desenvolvido para população clínica na Europa e validado para estudantes de graduação no Brasi ${ }^{19}$. Tal instrumento apresentou consistência interna adequada e confiabilidade teste-reteste em adultos brasileiros ${ }^{13,14}$. O questionário é composto por dez itens que mensuram a dificuldade em adormecer e sintomas de sonolência, as respostas são dadas em uma escala de sete pontos $(1=$ nunca; $2=$ muito raramente; $3=$ raramente; $4=$ às vezes; $5=$ fre- 
quentemente $; 6$ = muito frequentemente; 7 = sempre). Para fins de analise atribui-se uma pontuação derivada da somatória das respostas, sendo que, quanto maior a pontuação pior a qualidade do sono. Para este estudo, foi considerado AQS quando a pontuação foi de 10 a 24 pontos, e IQS quando a pontuação alcançou valor maior ou igual a 25 pontos $^{19}$. O número de vezes em que o participante foi classificado com alteração da QS ao longo dos anos com coleta de dados foi categorizado como segue: nenhuma alteração ao longo do seguimento e; uma ou mais alterações ao longo do seguimento.

Foi utilizado questionário para mensurar as seguintes variáveis: sexo (masculino e feminino), idade (numérico), etilismo (consumir bebidas alcoólicas com frequência de quatro ou mais vezes por semana); tabagismo (relatar o hábito de fumar ao menos um cigarro por dia) e a classe econômica por meio dos critérios da Associação Brasileira de Empresas de Pesquisa ${ }^{20}$, que leva em consideração para a classificação a somatória da pontuação (variando de 0 a 46 pontos) sendo 0 a classe econômica mais baixa (E) e 46 a classe econômica mais alta $(\mathrm{A} 1)^{20}$.

Foi verificada a circunferência de cintura, segundo protocolo de Lohman, Roche e Martorell21, o usuário ficou em posição ortostática com o abdômen relaxado e a fita foi colocada horizontalmente no ponto de menor circunferência entre a última costela e a crista ilíaca. Para tanto, utilizou-se uma trena antropométrica da marca Sanny. Foi considerado obesidade abdominal o adulto com a circunferência de cintura com $102 \mathrm{~cm}$ ou mais para homens e $88 \mathrm{~cm}$ ou mais para mulheres ${ }^{22}$.

A prática de atividade física foi avaliada por meio do questionário Baecke et al. ${ }^{23}$. Esse instrumento foi validado para população brasileira ${ }^{24} \mathrm{e}$ considera as atividades físicas praticadas nos seguintes domínios: ocupacional, lazer e locomoção. O questionário é composto por 16 perguntas com opções de resposta em escala tipo likert (nunca, raramente, algumas vezes, frequentemente e sempre). O calculo do escore foi realizado utilizando fórmula proposta por Baecke et al. ${ }^{23}$ e resultou no escore de atividade física total, posteriormente dividido em quartis: quartil 1 (insuficientemente ativo) quartil 2 e 3 (moderadamente ativo) e quartil 4 (ativo). Foram considerados insuficientemente ativos os adultos que estavam no quartil 1.

Para fins de análises descritivas, considerando a presença de normalidade das variáveis descritivas (testada pelo teste de Kolmogorov Smirnov) as mesmas foram apresentadas em através de média e desvio padrão.
Para verificar a diferença entre valores médios de idade, classe econômica, atividade física habitual, circunferência de cintura entre os adultos classificados com qualidade do sono adequada e inadequada, foi utilizado o teste t de Student para amostras independentes.

Para comparar a proporção de participantes com doenças (hipertensão arterial, infarto, diabetes e dislipidemias) entre os que tinham qualidade do sono adequada e inadequada foi utilizado o teste Qui-quadrado com correção de Yates. A regressão logística binária foi utilizada para associar QS (variável independente) com Doenças (variável dependente, deixando claro quem é $=0$ e quem é $=1$ ). Os potenciais fatores de confusão considerados na análise ajustada foram: sexo (categórico), idade (numérico), atividade física (numérico), obesidade abdominal (numérico), etilismo (categórico) e tabagismo (categórico). Na seleção das variáveis para compor o modelo ajustado foi foram mantidas no modelo as variáveis que são citadas pela literatura como potencias fatores de confusão $\mathrm{o}^{1,13-15,25}$, as variáveis foram inseridas simultaneamente no modelo, sem a necessidade de associação significativa no teste qui-quadrado. O teste de Hosmer-Lemeshow foi utilizado para analisar quão bem os dados estavam ajustados aos modelos multivariados. O nível de significância adotado $\mathrm{p}<$ 0,05 . Todas as análises foram realizadas no programa estatístico BioEstat (versão 5.0).

\section{Resultados}

A amostra foi composta por 169 adultos usuários do sistema público de saúde, não sendo registra nenhuma recusa por parte dos participantes. Porém, foram excluídos um total de 342 casos (122 por falta de interesse em continuar no seguimento, 119 por problema de saúde, 80 por mudança de endereço e telefone e 19 por óbito).

Quando comparados os participantes do grupo perda amostral $(n=342)$ com os participantes do presente estudo ( $n=169)$ não foram encontradas diferenças para a ocorrência de hipertensão arterial, dislipidemia, diabetes mellitus, infarto, nível de atividade física, obesidade, classe econômica, sexo e idade e qualidade do sono $(p>0,05)$. Foram analisados dados completos de 169 adultos, com média de idade 61,80 anos (desvio padrão igual 8,80 anos), sendo $68,6 \%$ do sexo feminino, $78,7 \%$ da classe econômica baixa (B2, C, D e E) e $83,4 \%$ com pelo menos uma alteração na QS. Verificou-se que $50,3 \%$ tinham obesidade abdominal e $26,6 \%$ encontravam-se no quartil 1 considerados com baixo nível de atividade física. 
Não foram encontradas diferenças significativas para idade, classe econômica, atividade física, circunferência de cintura, sexo tabagismo e etilismo entre os adultos com qualidade do sono IQS e AQS (Tabela 1).

Quando comparados o grupo IQS e AQS, não foram identificas diferenças significativas para a proporção de hipertensão arterial $(\mathrm{p}=0,642)$, infarto $(\mathrm{p}=$ $0,889)$ e dislipidemia $(\mathrm{p}=0,086)$. Porém, a proporção de adultos com DM foi estatisticamente maior no grupo IQS comparado ao AQS ( $\mathrm{p}=0,024)$ - Tabela 2 .

$\mathrm{Na}$ Tabela 3 estão os resultados da análise de regressão logística bruta e ajustada para associação entre IQS e AQS. $\mathrm{Na}$ análise bruta, a variável IQS se associou com a presença de diabetes mellitus. A associação se manteve significativa mesmo após ajuste, em que pessoas com IQS apresentam 3,4 vezes mais chances de reportarem diabetes mellitus (OR = 3,47; IC95\%: 1,06 -11,30) quando comparadas às com AQS (Tabela 3 ).

\section{Discussão}

Os resultados do estudo mostram que as pessoas com IQS apresentaram maiores chances de serem acometidas por diabetes mellitus comparadas as com AQS, mesmo após ajuste por fatores de confusão, como sexo, idade, atividade física, obesidade abdominal, etilismo e tabagismo.

No presente estudo, não foram observadas diferenças significativas de idade entre os adultos dos grupos IQS (63,43 anos) e AQS (61,43 anos). Esses achados corroboram ao de estudo prévio com amostra relativamente homogênea em relação à idade ${ }^{25}$. Em relação ao sexo também não foi observada diferença na comparação dos dois grupos (IQS vs. AQS). No entanto, tem sido observado que ser do sexo feminino é fator predisponente para a $\operatorname{IQS}^{26,27}$. Mulheres adultas são mais propensas a apresentar IQS devido às mudanças relacionadas ao período de pós-menopausa ${ }^{27}$.

Sabe-se que existe estreita relação entre IQS e as doenças cardiometabólicas ${ }^{7}$, visto que sono curto $(<6$ horas/noite) eleva em 1,22 vezes as chances de hipertensão autorreferida em adultos com 18 anos de idade ou mais ${ }^{7}$. Estudo de coorte que avaliou insônia e sono insatisfatório em 4.994 adultos com média de idade de 62,1 anos, verificou que tais distúrbios aumentaram em 29,0\% ( $R R=1,29$; IC95\%: 1,00 - 1,66) o risco de doenças cardiovasculares: infarto, angina, revascularização coronariana e acidente vascular encefálico, ao longo de 11,6 anos de acompanhamento ${ }^{26}$.

No entanto, o presente estudo identificou maiores chances de diabetes mellitus $(p<0,05)$ quando presen-
Tabela 1 - Características gerais da amostra de acordo com adequada ou inadequada qualidade do sono em adultos atendidos pelo Sistema Único de Saúde. Presidente Prudente, São Paulo, Brasil, 2015.

\begin{tabular}{|c|c|c|c|}
\hline \multirow{3}{*}{ Variáveis } & \multicolumn{2}{|c|}{ Qualidade do sono } & \multirow{3}{*}{ p-valor } \\
\hline & Adequada $(\mathrm{n}=28)$ & Inadequada $(\mathrm{n}=141)$ & \\
\hline & Média (DP) & Média (DP) & \\
\hline Idade (anos) & $63,43(10,85)$ & $61,35(8,32)$ & $0,344^{*}$ \\
\hline$\Sigma \mathrm{CE}$ & $19,07(5,54)$ & $19,77(4,96)$ & $0,504^{*}$ \\
\hline $\mathrm{AFH}$ (escore) & $7,12(1,57)$ & $6,96(1,37)$ & $0,581^{*}$ \\
\hline \multirow[t]{2}{*}{$\mathrm{CC}(\mathrm{cm})$} & $90,67(10,09)$ & $93,78(14,24)$ & $0,275^{*}$ \\
\hline & n (\%) & n (\%) & \\
\hline \multicolumn{4}{|l|}{ Sexo } \\
\hline Homem & $13(24,5)$ & $40(75,5)$ & \multirow{2}{*}{$0,097^{* *}$} \\
\hline Mulher & $15(12,9)$ & $101(87,1)$ & \\
\hline \multicolumn{4}{|l|}{ Tabagismo } \\
\hline Sim & $5(26,3)$ & $14(73,7)$ & \multirow{2}{*}{$0,376^{* *}$} \\
\hline Não & $23(15,3)$ & $127(84,7)$ & \\
\hline \multicolumn{4}{|l|}{ Etilismo } \\
\hline Sim & $25(15,4)$ & $137(84,6)$ & \multirow{2}{*}{$0,664^{* *}$} \\
\hline Não & $3(42,9)$ & $4(57,1)$ & \\
\hline
\end{tabular}

*Teste $t$ de Student independente; ** Qui-quadrado; DP = desvio padrão; $\Sigma \mathrm{CE}$ = soma da pontuação obtida no questionário da ABEP para classificação de classe econômica; $\mathrm{AFH}=$ atividade física; $\mathrm{CC}=$ circunferência de cintura.

Tabela 2 - Comparação da proporção de doenças cardiometabólicas entre os grupos de qualidade do sono adequada vs inadequada em adultos atendidos pelo Sistema Único de Saúde. Presidente Prudente, São Paulo, Brasil, 2015.

\begin{tabular}{|c|c|c|c|}
\hline \multirow{3}{*}{ Doenças } & \multicolumn{2}{|c|}{ Qualidade do sono } & \multirow{3}{*}{ p-valor* } \\
\hline & $\begin{array}{c}\text { Adequada } \\
(\mathrm{n}=28)\end{array}$ & $\begin{array}{c}\text { Inadequada } \\
(\mathrm{n}=141)\end{array}$ & \\
\hline & n (\%) & n (\%) & \\
\hline Hipertensão arterial & & & 0,642 \\
\hline Sim & $17(60,7)$ & $92(65,2)$ & \\
\hline Não & $11(39,2)$ & $49(34,8)$ & \\
\hline Infarto & & & 0,889 \\
\hline Sim & $2(7,2)$ & $14(10,1)$ & \\
\hline Não & $26(92,8)$ & $124(89,9)$ & \\
\hline Diabetes & & & 0,024 \\
\hline Sim & $3(10,8)$ & $47(33,3)$ & \\
\hline Não & $25(89,2)$ & $94(66,7)$ & \\
\hline Dislipidemia & & & 0,086 \\
\hline Sim & $11(39,2)$ & $81(57,4)$ & \\
\hline Não & $17(60,7)$ & $60(42,6)$ & \\
\hline
\end{tabular}

*Qui-quadrado com correção de Yates.

te IQS em comparação a AQS. Sabe-se que esse resultado ratifica a relação entre qualidade do sono e alterações metabólicas ${ }^{28}$. Em estudo envolvendo adultos de 45 a 64 anos de idade dos Estados Unidos, foi identificado que pessoas que apresentaram apneia obstrutiva do sono eram mais suscetíveis à presença do diabetes 
Tabela 3 - Regressão logística binária bruta e ajustada para associação entre qualidade do sono e doenças cardiometabólicas em adultos atendidos pelo Sistema Único de Saúde. Presidente Prudente, São Paulo, Brasil, 2015.

\begin{tabular}{|c|c|c|c|c|}
\hline & HÁ & Infarto & $\mathrm{DM}$ & DLP \\
\hline Variável & OR (IC95\%) & OR (IC95\%) & OR (IC95\%) & OR (IC95\%) \\
\hline \multicolumn{5}{|l|}{ Análise Bruta } \\
\hline QS adequada & 1,00 & 1,00 & 1,00 & 1,00 \\
\hline QS inadequada & $1,21(0,52-2,79)$ & $1,46(0,31-6,85)$ & $4,17(1,19-14,51)$ & $2,08(0,91-4,77)$ \\
\hline \multicolumn{5}{|l|}{ Análise Ajustada* } \\
\hline QS adequada & 1,00 & 1,00 & 1,00 & 1,00 \\
\hline QS inadequada & $1,18(0,69-2,01)$ & $1,42(0,31-6,56)$ & $3,47(1,06-11,30)$ & $1,53(0,80-2,91)$ \\
\hline
\end{tabular}

QS = qualidade do sono; OR = razão de chance; IC95\%; *análise ajustada por sexo, idade, atividade física, obesidade abdominal, etilismo e tabagismo, $\mathrm{IC}=$ intervalo de confiança de 95\%; HA = hipertensão arterial; DM = diabetes mellitus; $\mathrm{DLP}=$ dislipidemia.

mellitus $(p \leq 0,01)^{29}$. Problemas do sono podem estar relacionados com o metabolismo de liberação e resistência à insulina que, por sua vez, pode levar a graves quadros de diabetes mellitus tipo $2^{30}$. Do ponto de vista fisiológico, a privação do sono causa alterações que reduzem a sensibilidade à insulina, diminuem a tolerância à glicose e o metabolismo da mesma é prejudicado. Em conjunto, esses fatores colaboram para maior prevalência de diabetes mellitus em indivíduos com IQS ${ }^{28}$.

Assim como a obesidade, inatividade física não atuou como fator de confusão nos resultados. Entretanto, estudos anteriores indicaram relação direta entre menor prática de atividade física e $\mathrm{IQS}^{25}$. A inatividade física é vista como fator que eleva em 2,7 vezes as chances de diagnóstico de apneia obstrutiva do sono ${ }^{25}$, e os que praticavam exercícios com tempo superior a 1.200 minutos/semana apresentaram chances reduzidas de 40,0\% (OR = 0,6; IC95\%: 0,5 - 0,8) para este disgnóstico ${ }^{25}$.Esses resultados indicam que a prática de atividade física é um importante instrumento de enfrentamento para IQS, em direção a reduzir os problemas relacionados de saúde e a IQS.

Dentre as limitações deste estudo destacam-se: um possível viés de seleção da amostra. A perda amostral do estudo foi alta ( 67\% de perda amostral), sendo superior a prevista no cálculo amostral $(40,0 \%)$, ao final das cinco avaliações. Outra limitação deste estudo é que as qualidades psicométricas dos instrumentos utilizados não foram analisadas. Porém, os instrumentos foram validados para população brasileira ${ }^{19,25}$.

Cabe ressaltar que a pesquisa é decorrente de estudo longitudinal com 30 meses de seguimento, fato pouco observado em pesquisas nacionais que avaliam população atendida pela rede pública de saúde brasileira. Outro ponto forte deste estudo é que não houve diferenças significativas na ocorrência de doença e qualidade do sono entre os que concluíram o estudo e aqueles considerados perdas de seguimento.

Conclui-se que a inadequada qualidade do sono apresenta relação com a ocorrência de diabetes mellitus em adultos atendidos na atenção básica do Sistema Único de Saúde da cidade de Presidente Prudente, mesmo após ajuste para potenciais fatores de confusão, dentre os quais o nível de atividade física, obesidade abdominal, etilismo e tabagismo.

\section{Conflito de interesses}

Os autores declaram não haver conflito de interesses.

\section{Contribuições dos autores}

Ricardo SJ, participou de toda concepção do estudo. Araujo MYC, participou da coleta de dados, redação e revisão do estudo. Mantovani AM, participou da concepção inicial do estudo na busca de literatura e revisão, Santos LL participou da coleta de dados, da busca de literatura e concepção inicial da redação, Turi BC, participou na concepção inicial do estudo, na correção da redação e revisão critica do estudo, Queiroz DC, participou da coleta de dados e busca de literatura. Codogno JS, orientou e participou da concepção, revisão critica e correção de todo o estudo.

\section{Agradecimentos}

Ao apoio financeiro do Conselho Nacional de Desenvolvimento Científico e Tecnológico (CNPq), processo: 401178/2013-7 e a Coordenação de Aperfeiçoamento de Pessoal de Nível SuperiorBrasil (CAPES) - Código de Financiamento 001.

\section{Referências}

1. Lucassen EA, Piaggi P, Dsurney J, de Jonge L, Zhao X-c, Mattingly MS, et al. Sleep extension improves neurocognitive functions in chronically sleep-deprived obese individuals. PloS ONE. 2014;9(1):e84832.

2. João KADR, Jesus SN, Carmo C, Pinto P. The impact of sleep quality on the mental health of a non-clinical population. Sleep Med. 2018;46(1):69-73.

3. Kerkhof GA. Epidemiology of sleep and sleep disorders in The Netherlands. Sleep Med. 2017;(30):229-39. 
4. Adams RJ, Appleton SL, Taylor AW, Gill TK, Lang C, McEvoy RD, et al. Sleep health of Australian adults in 2016: results of the 2016 Sleep Health Foundation national survey. Sleep Health. 2017;(1):35-42.

5. Hirotsu C, Bittencourt L, Garbuio S, Andersen ML, Tufik S. Sleep complaints in the Brazilian population: Impact of socioeconomic factors. Sleep Sci. 2014;7(3):135-42.

6. Watson NF, Badr MS, Belenky G, Bliwise DL, Buxton OM, Buysse D, et al. Recommended amount of sleep for a healthy adult: a joint consensus statement of the American Academy of Sleep Medicine and Sleep Research Society. Sleep. 2015;38(6):843-4.

7. Grandner MA, Chakravorty S, Perlis ML, Oliver L, Gurubhagavatula I. Habitual sleep duration associated with self-reported and objectively determined cardiometabolic risk factors. Sleep Med. 2014;15(1):42-50.

8. Diaz K, Faverio P, Hospenthal A, Restrepo MI, Amuan ME, Pugh MJ. Obstructive sleep apnea is associated with higher healthcare utilization in elderly patients. Ann Thorac Med. 2014;9(2):92-8.

9. Leśniowska J, Schubert A, Wojna M, Baran IS, Fedyna M. Costs of diabetes and its complications in Poland. Eur J Health Econ. 2014;15(6):653-60.

10. Rosa MQ, Rosa RS, Correia MG, Araujo DV, Bahia LR, Toscano CM. Disease and Economic Burden of Hospitalizations Attributable to Diabetes Mellitus and Its Complications: A Nationwide Study in Brazil. Int J Environ Res Public Health. 2018;15(2):294.

11. Coutinho WF, Silva Júnior WS. Diabetes care in Brazil. Ann Glob Health. 2015;81(6):735-41.

12. Kerr M, Rayman G, Jeffcoate WJ. KERR. Cost of diabetic foot disease to the National Health Service in England. Diabet Med. 2014;(12):1498-504.

13. Zanuto EAC, Lima MCS, Araújo RG, Silva EP, Anzolin $\mathrm{CC}$, Araujo MYC et al. Distúrbios do sono em adultos de uma cidade do Estado de São Paulo. Rev Bras Epidemiol. 2015;18(1):42-53.

14. Morais LC, Zanuto EAC, Queiroz DC, Araújo MYC, Rocha APR, Codogno JS. Associação entre Distúrbios do Sono e Doenças Crônicas em Pacientes do Sistema Único de Saúde. J Phys Educ. 2017;28(1):e2844.

15. Zuo H, Shi Z, Yuan B, Dai Y, Hu G, Wu G, et al. Interaction between physical activity and sleep duration in relation to insulin resistance among non-diabetic Chinese adults. BMC Public Health. 2012;12(247).

16. IBGE. Censo demográfico: resultados preliminares: Presidente Prudente: 2017. ed. São Paulo. IBGE; 2017.

17. Kilsztajn S, Silva DF, Câmara MB, Ferreira VS. Grau de cobertura dos planos de saúde e distribuição regional do gasto público em saúde. Saúde e Soc. 2001;10(2):35-45.

18. Freitas Junior I, Castoldi RC, Moreti DG, Pereira ML, Cardoso ML, Codogno JS et al. Aptidão física, história familiar e ocorrência de hipertensão arterial, osteoporose, doenças metabólicas e cardíacas entre mulheres. Rev SOCERJ. 2009;22(3):158-64.
19. Falavigna A, de Souza Bezerra ML, Teles AR, Kleber FD, Velho MC, Silva RC, et al. Consistency and reliability of the Brazilian Portuguese version of the Mini-Sleep Questionnaire in undergraduate students. Sleep Breath. 2010;15(3):351-5.

20. ABEP- Associação Brasileira de Empresas de pesquisa. Critério de classificação econômica Brasil [internet]. São Paulo: ABEP, 2008. [citado em 2018 jun 30]. Disponível em: http://www.abep.org/.

21. Lohman TG, Roche AF,Martorell R, editors. Anthropometric standardization reference manual. Champaign (IL): Human Kinetics; 1988;39-54.

22. Expert Panel on Detection, Evaluation, and Treatment of High Blood Cholesterol in Adults. Executive Summary of The Third Report of The National Cholesterol Education Program (NCEP) Expert Panel on Detection, Evaluation, and Treatment of High Blood Cholesterol in Adults (Adult Treatment Panel III). JAMA. 2001;285(19):2486-97.

23. Baecke JA, Burema J, Frijters JE. A short questionnaire for the measurement of habitual physical activity in epidemiological studies. Am J Clin Nutr. 1982;36:936-42.

24. Florindo AA, Latorre MRDO. Validation and reliability of the Baecke questionnaire for the evaluation of habitual physical activity in adult men. Rev Bras Med Esporte. 2003;9(3):129-35.

25. Simpson L, McArdle N, Eastwood PR, Ward KL, Cooper $\mathrm{MN}$, Wilson AC, et al. Physical inactivity is associated with moderate-severe obstructive sleep apnea. J Clin Sleep Med. 2015;11(10):1091-9.

26. Bertisch SM, Pollock BD, Mittleman MA, Buysse DJ, Bazzano LA, Gottlieb DJ, et al. Insomnia with objective short sleep duration and risk of incident cardiovascular disease and all-cause mortality: Sleep Heart Health Study. Sleep. 2018;41(6).

27. Moudi A, Dashtgard A, Salehiniya H, Katebi MS, Razmara RM, Jani MR. The relationship between healthpromoting lifestyle and sleep quality in postmenopausal women. Biomedicine. 2018;8(2):11.

28. Briançon-Marjollet A, Weiszenstein M, Henri M, Thomas A, Godin-Ribuot D, Polak J. The impact of sleep disorders on glucose metabolism: endocrine and molecular mechanisms. Diabetol Metab Syndr. 2015;(7):25.

29. Nagayoshi M, Punjabi NM, Selvin E, Pankow JS, Shahar $\mathrm{E}$, Iso H, et al. Obstructive sleep apnea and incident type 2 diabetes. Sleep Med. 2016;25:156-61.

30. Reutrakul S, Van Cauter E. Sleep influences on obesity, insulin resistance, and risk of type 2 diabetes. Metabolism. 2018;84:56-66.

Recebido: 01/02/2019

Aprovado: 30/09/2019 